\title{
Host Galaxies of X-shaped Radio Sources
}

\author{
Alessondra Springmann \\ Office of Science, Science Undergraduate Laboratory Internship (SULI) \\ Wellesley College \\ Stanford Linear Accelerator Center \\ Stanford, CA
}

August 25, 2006

Prepared in partial fulfillment of the requirements of the Office of Science, Department of Energy's Science Undergraduate Laboratory Internship under the direction of Chi C. Cheung at the Kavli Institute for Particle Astrophysics and Cosmology, Stanford Linear Accelerator Center.

Participant:

Signature

Research Advisor:

Signature 


\section{TABLE OF CONTENTS}

$\begin{array}{ll}\text { Abstract } & \text { ii }\end{array}$

$\begin{array}{ll}\text { Introduction } & 1\end{array}$

$\begin{array}{ll}\text { Materials and Methods } & 3\end{array}$

$\begin{array}{ll}\text { Results } & 4\end{array}$

$\begin{array}{ll}\text { Discussion and Conclusions } & 5\end{array}$

$\begin{array}{lr}\text { Acknowledgments } & 7\end{array}$

$\begin{array}{lr}\text { References } & 7\end{array}$ 


\begin{abstract}
Host Galaxies of X-shaped Radio Sources. ALESSONDRA SPRINGMANN (Wellesley College, Wellesley, MA 02481) CHI C. CHEUNG (Kavli Institute for Particle Astrophysics and Cosmology, Stanford Linear Accelerator Center, Menlo Park, CA 94025).
\end{abstract}

The majority of radiation from galaxies containing active galactic nuclei (AGNs) is emitted not by the stars composing the galaxy, but from an active source at the galactic center, most likely a supermassive black hole. Of particular interest are radio galaxies, the active galaxies emitting much of their radiation at radio wavelengths. Within each radio galaxy, an AGN powers a pair of collimated jets of relativistic particles, forming a pair of giant lobes at the end of the jets and thus giving a characterisitic double-lobed appearance. A particular class of radio galaxies have an "X"-shaped morphology: in these, two pairs of lobes appear to originate from the galactic center, producing a distinctive X-shape. Two main mechanisms have been proposed to explain the X-shape morphology: one being through the merger of a binary supermassive black hole system and the second being that the radio jets are expanding into an asymmetric medium. By analyzing radio host galaxy shapes, we probe the distribution of the stellar mass to compare the differing model expectations regarding the distribution of the surrounding gas and stellar material about the AGN. 


\section{INTRODUCTION}

Among the largest and most prevalent structures in the Universe are extragalactic radio sources, emitting strongly in the radio portion of the spectrum. Thousands of these objects exist, ranging in size from approximately 50 kiloparsecs across (approximately 150,000 light-years) to 100 kiloparsecs (300 light-years). These radio sources are composed of relativistic plasma jets traveling at high relativistic speeds, and emit synchrotron radiation (relativistic electrons moving through weak magnetic fields). Extragalactic radio sources contain a central host galaxy, from which two jets are emitted. As the jets interact with the surrounding intergalactic medium, a pair of giant radio lobes form. Figure 1 shows Cygnus A, a typical extragalactic radio source approximately 100 kiloparsecs across, which exhibits a central host galaxy (blue), collimated jets, and radio lobes (both red).

In addition to being large, coherent structures, extragalactic radio sources are also highly energetic. The energy output of typical extragalactic radio sources is $10^{44} \mathrm{ergs} / \mathrm{second}$, with some sources having energies of up to $10^{59} \mathrm{ergs} / \mathrm{second}$. (Our Sun, in comparison, has a luminosity of $10^{33} \mathrm{ergs} / \mathrm{second}$, making it $10^{11}$ orders of magnitude less energetic than Cygnus A.) The majority of radio galaxies emit the majority of their radiation not from the stars, gas, and dust composing the galaxy, but from the active source at the center, an active galactic nuclei (AGN), widely believed to be a supermassive black hole [1].

Thousands of these extragalactic radio sources resemble the canonical object Cygnus A in that they posses one pair of radio jets. However, approximately a dozen radio galaxies have two sets of jets emitting from the central supermassive black hole, forming a distinctive "X"-shape [2]. Over the past three decades, the number of known sources possessing the X-shaped morphology has grown from a dozen to over a hundred candidates, allowing for more detailed studies of these objects, particularly regarding their shape and origin. Figures 2 and 3 show double radio sources J1513+2607 and J1606+0000, which display a distinctive 
X-shaped morphology.

Astronomers propose that this distinct shape may be the result of a recent collision or merger between two supermassive black holes, which can produce the extra set of jets and lobes. Another explanation is that the main jets expanded into an asymmetric medium, which we probe by studying the host galaxy. Hydrodynamical simulations by [3] (Figure 4) show that as a jet, aligned parallel to the semi-major axis of an elliptical galaxy ${ }^{1}$, propagates into the surrounding gas distribution, two major jets form, and smaller wings will form orthogonal to the major jets, producing X-shaped radio lobes.

From the x-ray observations performed by [4] of the gas surrounding elliptical galaxies, we know that stellar light is an efficient way of probing gas distribution, as the distribution of gas around a host galaxy follows the distribution of stars in the galaxy. Thus, a galaxy with an elliptical distribution of stars will have an elliptical distribution of surrounding gas. Wing formation is most pronounced in elliptical galaxies, with the main set of jets forming parallel to the major axis of the galaxy. Studying a small set of host galaxies, [3] found that more elliptical galaxies have a secondary set of "wings", which align orthogonal to the semi-major axis of the host galaxy, as shown in their simulations and in Figure 6.

In this paper, we investigate the ellipticity of the galaxies that play host to the Xshaped sources, in order to determine if galaxies with more elliptical distributions of gas have X-shaped jets. Using the ellipse package for Image Reduction and Analysis Facility $(\mathrm{IRAF})^{2}$ to model the elliptically-shaped isophotes, or regions of equal brightness, of the galaxies, we determined the ellipticity and extent of the host galaxies and compared these to radio galaxy morphology, and therefore tested the results of previous studies (such as those carried out by [3]) with a larger sample of X-shaped radio sources.

\footnotetext{
${ }^{1}$ An elliptical galaxy is an ellipsoid, or a three-dimensional ellipse, as shown in Figure 5. Viewed from Earth, it appears as an ellipse in the sky.

${ }^{2}$ IRAF is distributed by the National Optical Astronomy Observatories, which are operated by the Association of Universities for Research in Astronomy, Inc., under cooperative agreement with the National Science Foundation.
} 


\section{MATERIALS AND METHODS}

Our data of 19 host galaxies of X-shaped extragalactic radio sources were collected by the Sloan Digitized Sky Survey (SDSS). The images are 56-second exposures taken in the r-band filter, which has a central wavelength of 6280 ångstroms. All objects imaged are 18th magnitude ${ }^{3}$ or brighter in the $r$-band, as dimmer objects would otherwise not be sufficiently resolved by the SDSS charge-coupled device (CCD). The pixel scale of the SDSS CCD is 0.4 arcseconds and the seeing for each image is approximately one arcsecond. A sample source image, the host galaxy of source J0813+4347, is shown in Figure 7.

Elliptical isophotes, or regions of equal brightness, of these host galaxies were fitted by the ellipse routine of the Space Telescope Science Data Analysis System (STS-DAS) ${ }^{4}$ isophote package for IRAF.

The isophote fitting methods are described by [5]: the center and length of the outermost elliptical isophote's semi-major axis are specified by the user, then the software plots successively smaller isophotes on the image, as seen in Figure 8. Information pertaining to the isophote plots, such as the semi-major axis of the isophote in pixels, ellipticity of the isophote ${ }^{5}$, and position angle of the isophote relative to north in the image are written to a table.

A routine called bmodel then converts this table to a model of relative isophotal intensities and sizes, which can then be subtracted from the original image to judge the goodness of the isophote fits. Figure 9 shows the isophote model subtracted from the background image and that the isophote model matches the intensity of the host galaxy, leaving minimal residual background on the original image.

\footnotetext{
${ }^{3}$ The magnitude scale is a logarithmic scale that describes the relative brightness of stars, with the star Vega ( $\alpha$ Lyrae) defined as having magnitude 0.

${ }^{4}$ STS-DAS is a product of the Space Telescope Science Institute, which is operated by theAssociation of Universities for Research in Astronomy, Inc. for the National Aeronautics and Space Administration.

${ }^{5}$ Ellipticity is defined as $f=1-a / b$, where $a$ is the semi-major axis of the ellipse, and $b$ is the semi-minor axis of the ellipse. An ellipse with $f=0$ is a circle.
} 
The position angles of the radio lobes and wings of the actual X-shaped sources were measured from data taken by the Very Large Array (VLA) ${ }^{6}$. The difference between the position angles of the host galaxies' semi-major axes and the position angles of the radio wings of the X-shaped sources are shown in Table 1 with the values for the ellipticities of host galaxies, calculated from the isophote fits calculated from the ellipse routine.

\section{RESULTS}

Galaxy parameters (position angle of the main set of radio jets, position angle of the radio wings, position angle of the host galaxy in the optical, ellipticity of the host galaxy, and offset between the radio wing position angle and the optical host galaxy position angle) were measured for four known X-shaped sources from the literature [2] and five new objects found by Cheung with a distinct X-shape [2].

The distribution of host galaxy ellipticities for our 19 sources (in blue) is compared to a sample of "normal" (not X-shaped) host galaxies of extragalactic radio sources analyzed by [3] (white) is shown in Figure 10. Some of these host galaxies are highly elliptical; however, approximately eight of these objects have ellipticities consistent with the host galaxy being circular. Capetti found that the host galaxies of X-shaped sources tended to be highly elliptical [3], which is contrary to our finding of the host galaxies of these X-shaped objects ranging from being circular to highly elliptical. It appears that the host galaxy need not be highly elliptical to cause wing formation in these eight cases.

Although the ellipticities of these host galaxies show no specific trend toward either high or low ellipticity, the offset between the host galaxy optical semi-major axis position and that of the radio wings tends to be approximately orthogonal for six out of nine objects for which exist VLA observations, as shown in Figure 11.

With the position angles of the radio lobes and wings taken from the VLA data in

\footnotetext{
${ }^{6}$ VLA data courtesy of the National Radio Astronomy Observatory/Associated Universities, Inc.
} 
Table 1, there appears to be little connection between the ellipticity of the host galaxy and the presence of radio wings orthogonal to the semi-major axis of the host galaxy. This is evidenced by objects J0805+2409, J0831+3219, and J1614+2817, with low ellipticities (less than 0.12 ) and an approximately orthogonal offset between the radio wings and optical semi-major axis of the host galaxy.

However, we can only measure host galaxy position angles if they are highly elliptical (for ellipticities greater than 0.12), which produces low-confidence measurements of the isophotal position angles of the semi-major axes of the host galaxies.

Comparing the host galaxy position angle to the wing position angle, we find that the difference in these angles are clustered around $90^{\circ}$, or that the position angles are orthogonal, consistent with the findings of [3]. However, unlike [3], we find that galaxies with circular isophotes play host to X-shaped radio sources.

\section{DISCUSSION AND CONCLUSIONS}

We measured host galaxy ellipticities from 19 sources and these are plotted in Figure 10. Some of these host galaxies are highly elliptical, with ellipticity values of over 0.12 ; however, four of these objects have ellipticities consistent with being circular. We compared the ellipticities to the comparison sample of normal radio galaxies from [3] and the distributions are indistinguishable, contrary to the findings of [3]. Simulations performed by [3] that an elliptical distribution of gas about a host galaxy produces the main lobes and auxiliary wings seen in X-shaped sources. The host galaxy need not be highly elliptical to cause wing formation in extragalactic radio sources.

X-ray observations performed by [4] of 3C 403, an X-shaped source, demonstrating that the optical position angle of the host galaxy follows the X-ray gas distribution. Therefore, in at least one X-shaped source, we know that the gas follows the star distribution of 
the galaxy, and thus if the galaxy appears elliptical in the optical band, it is also most likely elliptical in its gas distribution. According to [3], if the stellar light follows the distribution of gas around the galaxy, is elliptical, and is on a scale much larger than that of the host, comparable to the size of the X-shaped lobes and wings, then the double morphology of the $\mathrm{X}$-shaped radio galaxies will form as the main set of jets expand into the surrounding gas.

Although the model proposed by [3] does not account for circular host galaxies, a way to resolve this discrepancy is to consider the orientation effects of the host galaxy. A galaxy with the form of an ellipsoid viewer from its most circular side will appear to have circular isophotes as a consequence of the observer's position. However, it is unlikely that galaxy having the shape of a spheroid (an ellipsoid with two axes equal in length) would have a spherical side facing Earth. It is more likely that, although our results for J0831+3219 agree with deeper observations by [6], the data from the Sloan Digital Sky Survey is "shallow", or that the objects were not sufficiently exposed to obtain a high enough signal to noise ration in order to resolve the elliptical isophotal structure of these host galaxies.

We compared our measurements with much deeper data taken at longer exposures for one well-studied, low-ellipticity object, J0831+3219 [6]. As our values for ellipticity and position angle of the host galaxy are consistent with Ulrich's, this demonstrates that the Sloan Digital Sky Survey data is valid and comparable to "deeper", or longer-exposure observations.

The model proposed by [3] might have difficulty explaining the circular hosts of these X-shaped sources, however, it should not be discounted. There are a number of papers, including [3], which describe hydrodynamical situations that lead to X-shaped source formation and are supported by not only with data but by simulations. Other models exist that propose that the formation of secondary wings in extragalactic radio sources due to the realignment of the central supermassive black hole of a host galaxy, due perhaps to galactic mergers, but they lack significant simulations or data to reinforce their predictions. In order 
to verify whether the Capetti or black hole realignment model most accurately describes the creation of X-shaped extragalactic radio sources and further understand the formation of these objects, more simulations of galactic mergers should be performed, in addition to deeper observations being made of both the host galaxies and X-shaped sources.

\section{ACKNOWLEDGMENTS}

This research was conducted at the Kavli Institute for Particle Astrophysics and Cosmology at the Stanford Linear Accelerator Center. I thank the U. S. Department of Energy, Office of Science and Michael Woods for the opportunity to participate in the SULI program and for the tremendous learning and research experience. Immense thanks are due to my mentor Teddy Cheung for his knowledge of extragalactic radio astronomy, patience, persistence, and sense of humor. I also thank Alexandra Rahlin for providing invaluable help with Matlab scripting and $\mathrm{H}_{\mathrm{E}} \mathrm{X}$ formatting. I am grateful to the faculty and staff of KIPAC, especially Stuart Marshall and Grzegorz Madejski, for hosting me this summer and generously providing us students with a wealth of resources and encouragement.

\section{REFERENCES}

[1] D. S. De Young, The Physics of Extragalactic Radio Sources, 1st ed. Chicago, Illinois: The University of Chicago Press, 2002, vol. 1.

[2] C. C. Cheung, "First 'Winged' and 'X'-shaped radio sources," Astronomy Journal, 2006 (submitted).

[3] A. Capetti, S. Zamfir, P. Rossi, G. Bodo, C. Zanni, and S. Massaglia, "On the origin of X-shaped radio-sources: New insights from the properties of their host galaxies," Astronomy \&3 Astrophysics, vol. 394, pp. 39-45, Oct. 2002. 
[4] R. P. Kraft, M. J. Hardcastle, D. M. Worrall, and S. S. Murray, "A Chandra Study of the Multicomponent X-Ray Emission from the X-shaped Radio Galaxy 3C 403," The Astrophysical Journal, vol. 622, pp. 149-159, Mar. 2005.

[5] R. I. Jedrzejewski, "CCD surface photometry of elliptical galaxies. I - Observations, reduction and results," Monthly Notices of the Royal Astronomical Society, vol. 226, pp. 747-768, June 1987.

[6] M.-H. Ulrich and J. Roennback, "The host of B2 0828+32, a radio galaxy with two sets of radio lobes," Astronomy \& Astrophysics, vol. 313, pp. 750-754, Sept. 1996.

[7] J. P. Leahy, G. G. Pooley, and J. M. Riley, "The polarization of classical double-radio sources," Monthly Notices of the Royal Astronomical Society, vol. 222, pp. 753-785, Oct. 1986.

[8] J. Dennett-Thorpe, P. A. G. Scheuer, R. A. Laing, A. H. Bridle, G. G. Pooley, and W. Reich, "Jet reorientation in active galactic nuclei: two winged radio galaxies," Monthly Notices of the Royal Astronomical Society, vol. 330, pp. 609-620, Mar. 2002. 


\section{TABLES}

Table 1: Object name (in J2000 coordinates), the position angle of the main set of radio jets, the position angle of the radio wings, the position angle of the host galaxy in the optical, the ellipticity of the host galaxy from the optical images, and the offset between the radio wing position angle and the optical host galaxy position angle are shown.

\begin{tabular}{|c|c|c|c|c|c|}
\hline Object Name & Radio Lobe PA & Radio Wing PA & Optical PA & Optical Ellipticity & Offset \\
\hline \hline J0805+2409 & 123 & 58 & 140 & 0.013 & 82 \\
J0813+4347 & 105 & 140 & 40 & 0.137 & 100 \\
J0831+3219 & 60 & 177 & 76 & 0.085 & 101 \\
J1005+1154 & 170 & 125 & 102 & 0.102 & 23 \\
J1020+4831 & 170 & 125 & 134 & 0.156 & 9 \\
J1130+0058 & 100 & 10 & 35 & 0.249 & 25 \\
J1513+2607 & 12 & 130 & 33 & 0.341 & 97 \\
J1606+0000 & 55 & 23 & 113 & 0.418 & 90 \\
J1614+2817 & 106 & 175 & 82 & 0.104 & 93 \\
\hline
\end{tabular}




\section{FIGURES}

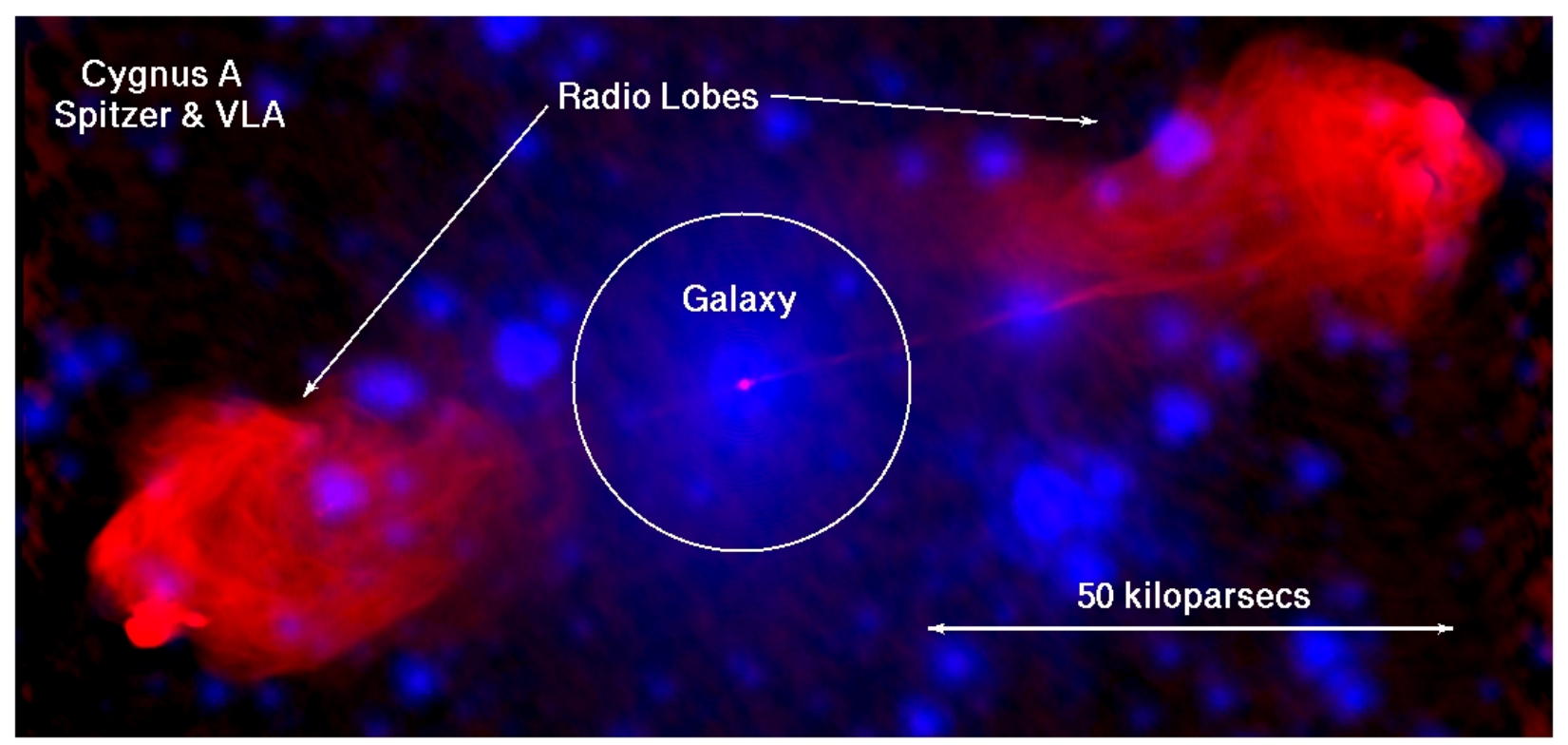

Figure 1: Extragalactic radio source Cygnus A. The host galaxy is imaged in the optical band shown in blue at the center, while the radio jets and lobes are shown in red. The total size of Cygnus A is 100 kiloparsecs across, approximately 300,000 light-years. The optical image of the host galaxy is courtesy of the Spitzer Space Telescope and the radio image was taken with the Very Large Array, courtesy of the National Radio Astronomy Observatory/Associated Universities, Inc. 


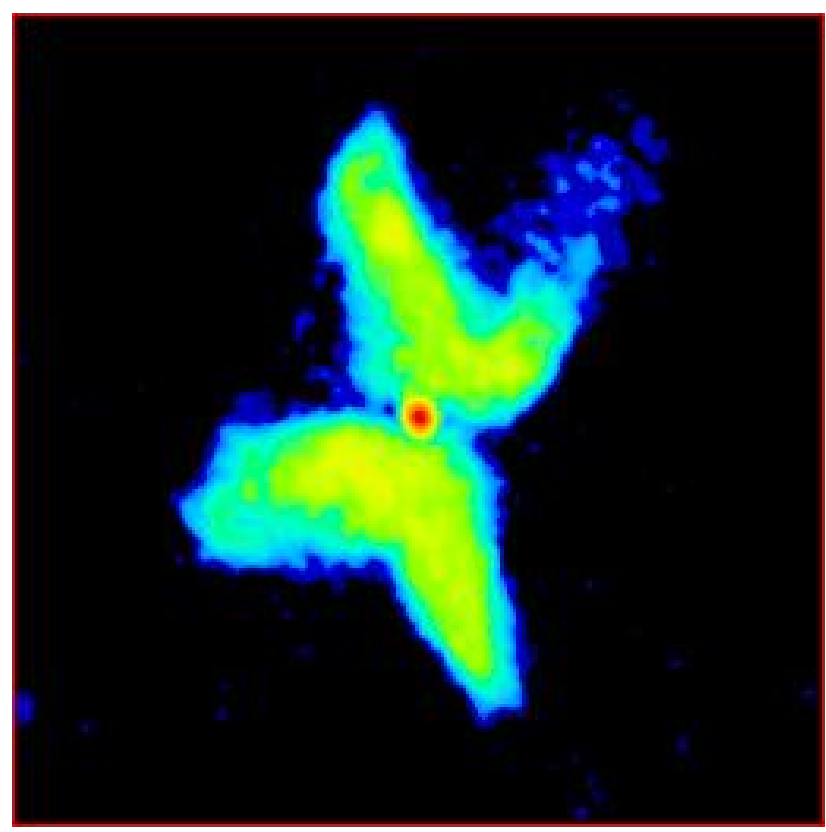

Figure 2: J1513+2607, an extragalactic radio source with two pairs of jets, from [7].

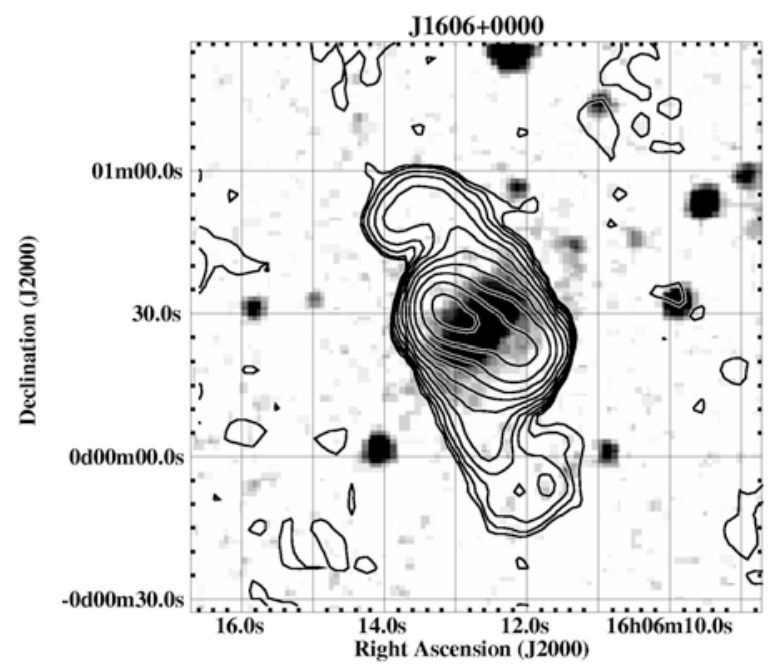

Figure 3: J1606+0000 in the radio band, showing two sets of jets in a winged shape, from $[2]$. 

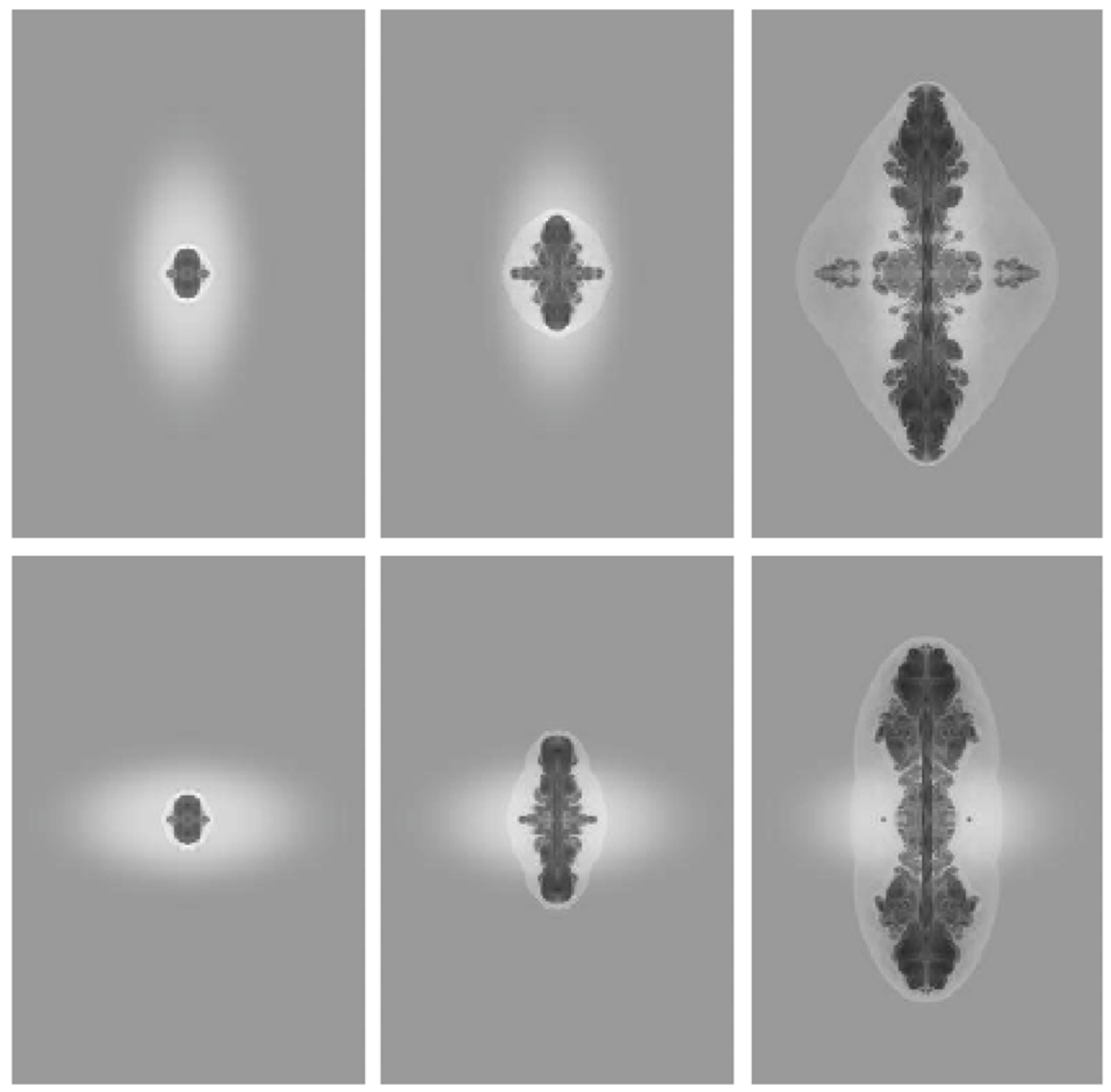

Figure 4: Hydrodynamical simulations performed by [3]. These numerical simulations show density images representing the evolution of the radio-source in the stratified medium of the host galaxy. The top panels refer to the case when the jet is oriented parallel to the major axis of the gas distribution, while the lower panels to the case when the jets is parallel to the minor axis. The white represents the distribution of gas about the host galaxy, while the dark gray represents the expanding radio jets. 


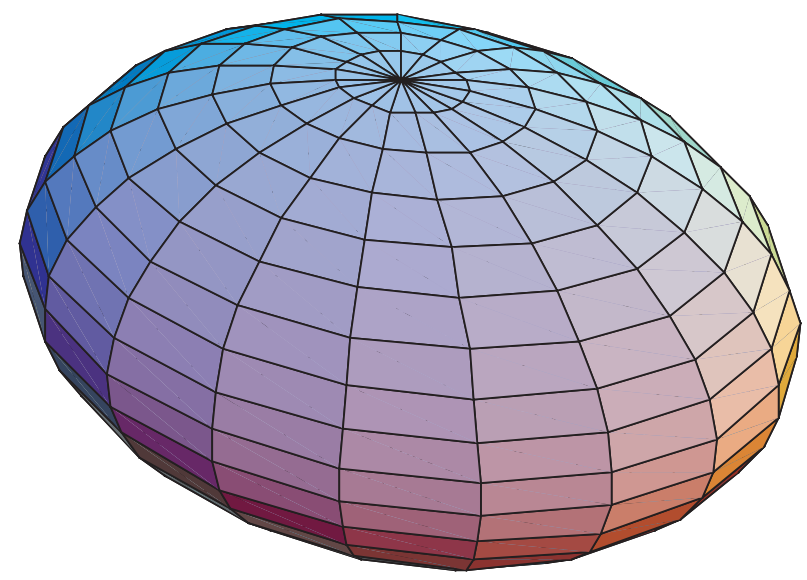

Figure 5: A model of an elliptical galaxy as a three-dimensional ellipse, or ellipsoid. When viewed from Earth it appears to be a flat ellipse.

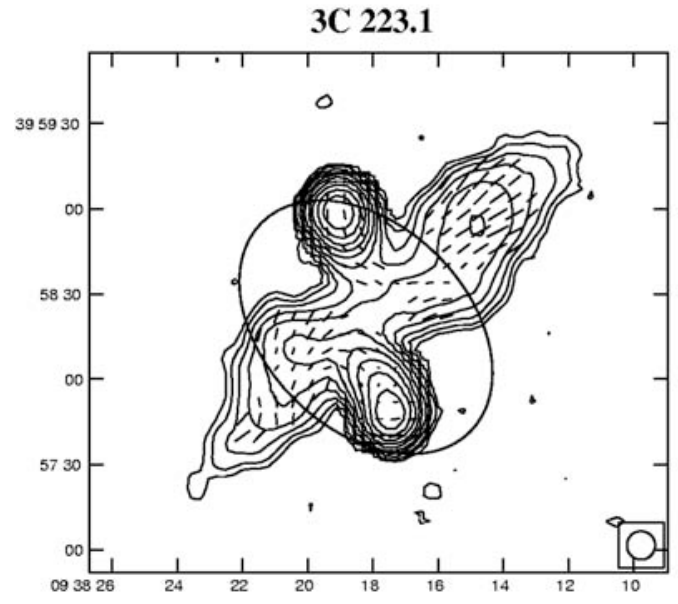

Figure 6: Superposition of the host galaxy shape (not in scale) onto the radio image maps for X-shaped source 3C 223.1. Superposition by [3], with radio maps from [8]. 


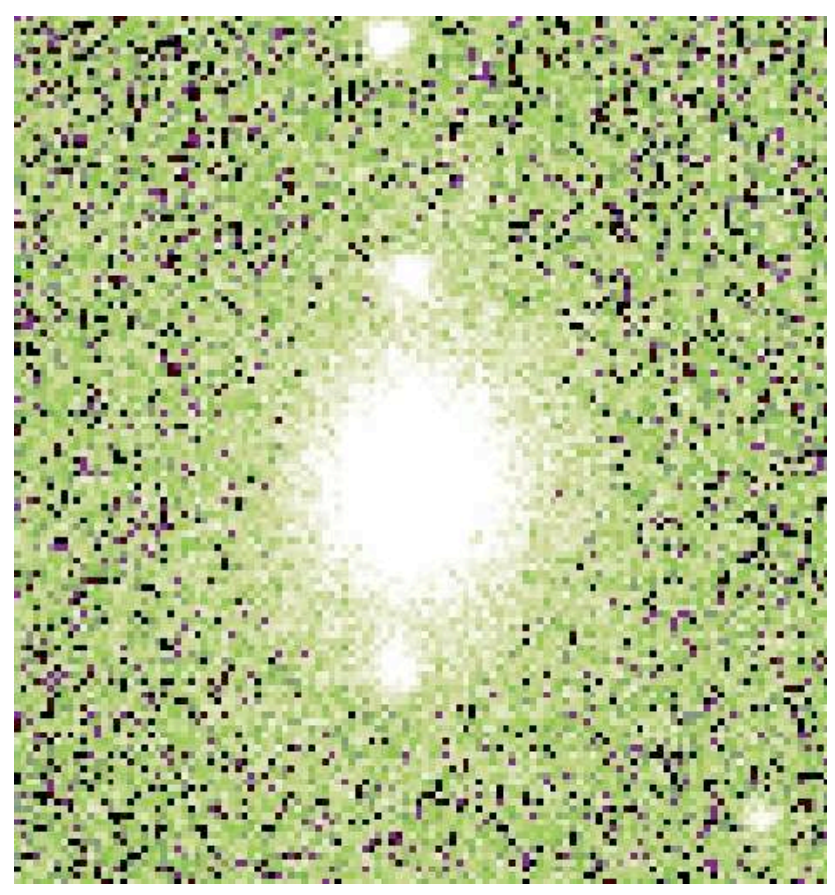

Figure 7: Detail of J0813+4347, an elliptical host galaxy imaged in the $r$-band by the SDSS telescope.

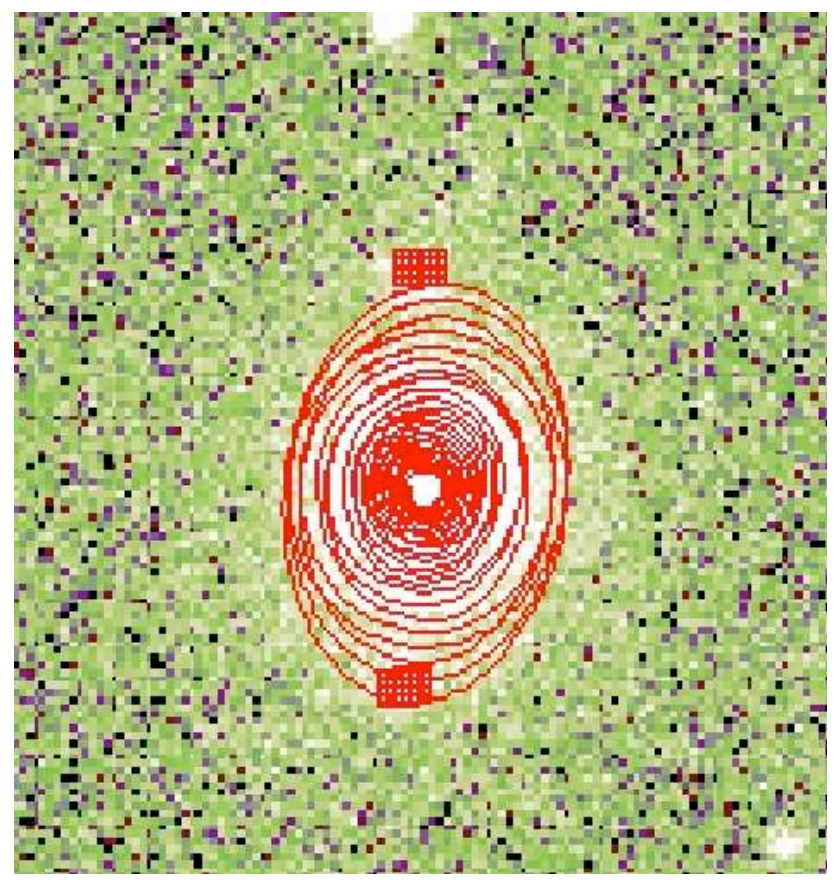

Figure 8: Isophotes fitted to J0813+4347 by the ellipse routine of the SDS-DAS package for IRAF. 


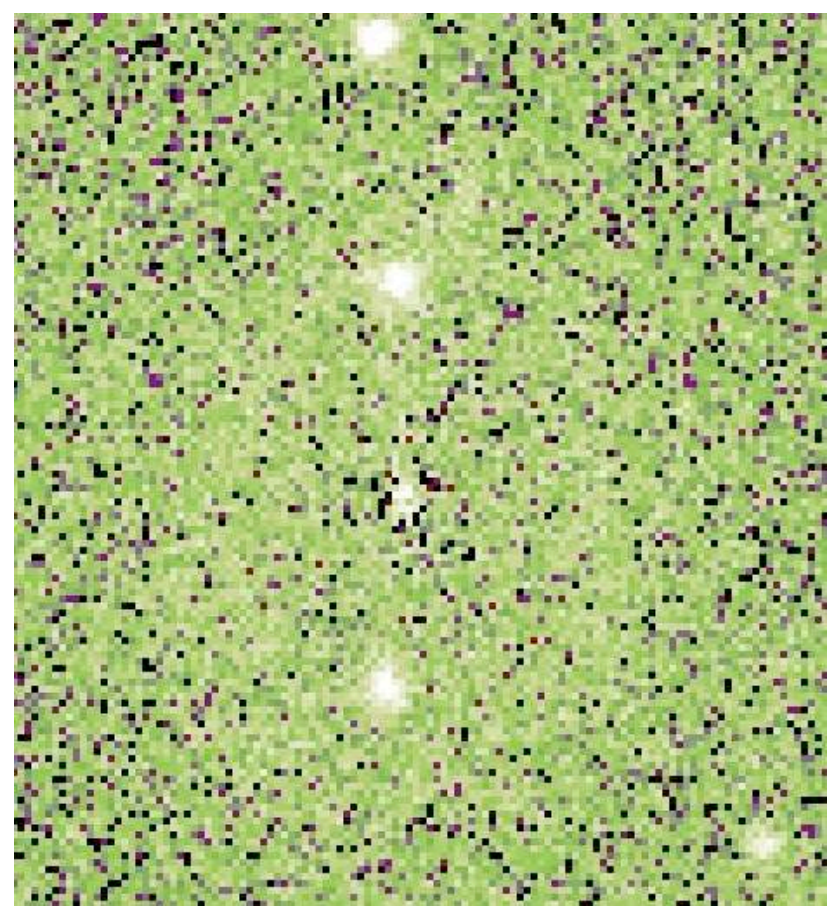

Figure 9: The J0813+4347 image with the isophote model subtracted from the data image. The original galaxy has been completely removed, except for the bright point at the center (the host galaxy AGN).

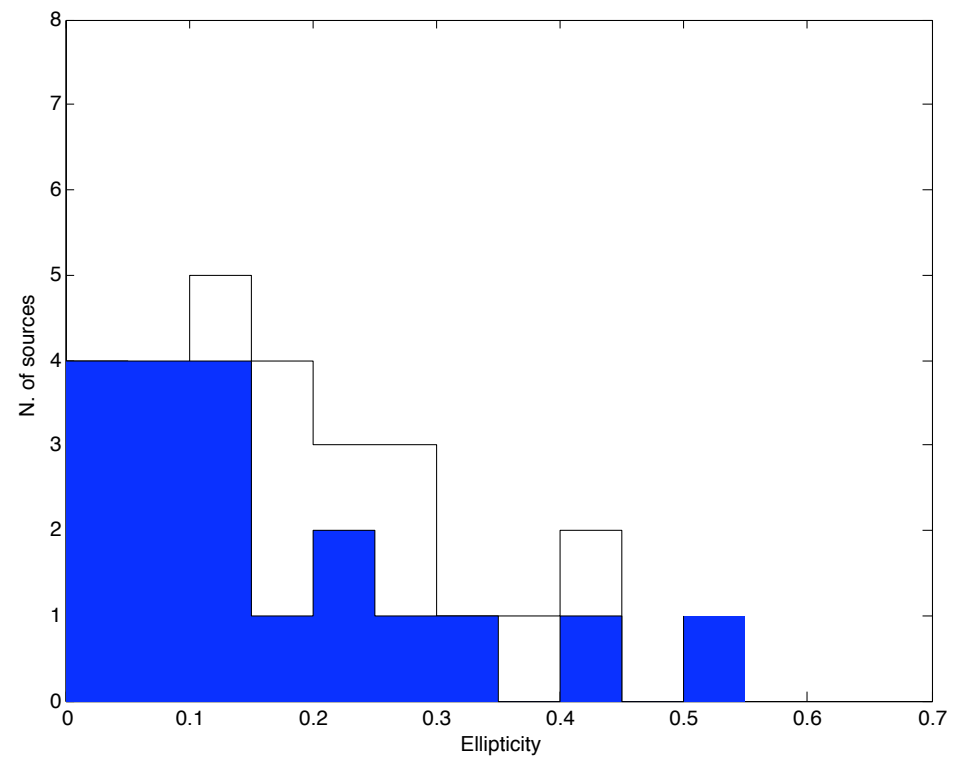

Figure 10: A histogram of the ellipticities of the host galaxies of X-shaped sources. The white histogram are the "control" objects from [3] and the blue histogram are the host galaxies of $\mathrm{X}$-shaped radio sources. 


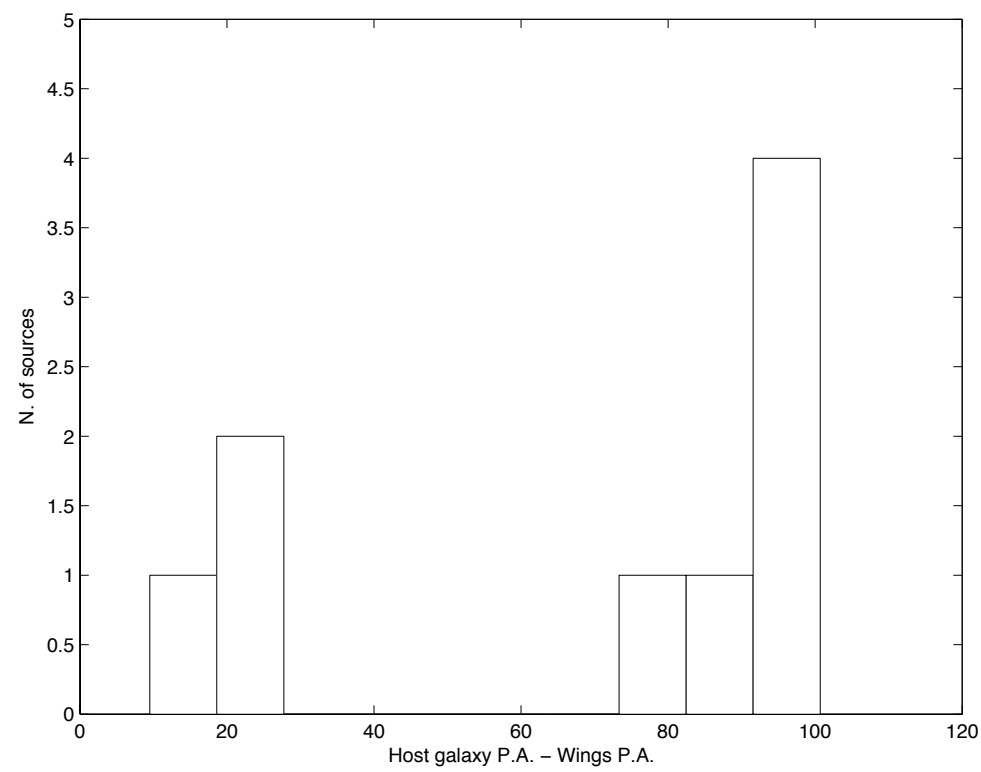

Figure 11: A histogram of the position angle offsets between the semi-major axis of the host galaxies and radio wings of the $\mathrm{X}$-shaped radio sources. 